\section{Cahiers de Narratologie}

Analyse et théorie narratives

40 | 2021

École, Récits, Nation

\title{
Instruction artistique et éducation esthétique au cours de la période fasciste
}

\section{Stefano Lentini}

\section{(2) OpenEdition}

\section{Journals}

Édition électronique

URL : https://journals.openedition.org/narratologie/12600

DOI : 10.4000/narratologie. 12600

ISSN : 1765-307X

Éditeur

LIRCES

Référence électronique

Stefano Lentini, «Instruction artistique et éducation esthétique au cours de la période fasciste », Cahiers de Narratologie [En ligne], 40 | 2021, mis en ligne le 09 décembre 2021, consulté le 03 janvier 2022. URL : http://journals.openedition.org/narratologie/12600; DOI : https://doi.org/10.4000/ narratologie. 12600

Ce document a été généré automatiquement le 3 janvier 2022.

Article L.111-1 du Code de la propriété intellectuelle. 


\title{
Instruction artistique et éducation esthétique au cours de la période fasciste
}

\author{
Stefano Lentini
}

1 Après la « Grande Guerre » et les graves échecs qu'elle occasionna, comme la défaite de Caporetto ou la débâcle de l'Italie aux Conférences de la paix de Versailles, on imputa une part de la responsabilité des insuccès rencontrés à la «mauvaise qualité de l'école ». En particulier, on accusait l'école italienne d'avoir consacré peu d'attention à la formation d'une solide conscience nationale, de dispenser une formation démagogique et pro-démocratique qui n'était que superficiellement supranationale, et de ne pas avoir été capable de former une classe dirigeante adaptée, susceptible de réaliser un virage moral dans le Beau pays ${ }^{1}$.

Dans un climat ambigu et incertain, entre idéalisme et autoritarisme, Benedetto Croce et Giovanni Gentile se retrouvèrent tous deux dans les groupes qui accompagnaient Benito Mussolini, le chef de file du fascisme ${ }^{2}$, pour concevoir et développer une réforme de l'école ${ }^{3}$. La nomination de Gentile au ministère de l'Instruction Publique représenta une décision habile de la part de Mussolini pour s'assurer l'appui d'autres intellectuels influents, comme Ernesto Codignola, Giuseppe Lombardo Radice, Vito Fazio-Allmayer, auxquels s'ajoutait un nombre important d'enseignants qui, aux côtés du philosophe de Castelvetrano, appartenaient au Fascio de l'Éducation Nationale créé en $1920^{4}$.

Définie par Benito Mussolini comme la "plus fasciste des réformes", la réforme de l'école de Gentile promulguée en 1923 fut en réalité fasciste dans la forme, mais libérale sur le fond ${ }^{5}$. Cette réforme de droite, libérale et bourgeoise, «se propose de redonner de la rigueur aux études et de la fermeté à la tradition de l'école humaniste qui était déjà à l'origine de la loi Casati, et de développer, tout spécialement à l'école élémentaire, les principes de la pédagogie idéaliste. La réforme insistait largement sur la méthode autoritaire dans l'organisation et le système de l'école ${ }^{6}$ ", ce qui passait par une réorganisation décisive de tous les niveaux d'enseignement, de l'école maternelle à l'université. Le but était de s'écarter du modèle de l'école sélective et aristocratique, 
pensée pour former les futures classes dirigeantes ${ }^{7}$. S'inspirant du projet de la loi Casati de 1859, " la loi de 1923 se fondait sur le caractère central et supérieur de la culture classique et humaniste enseignée au lycée et sur le caractère subalterne de la culture technique et professionnelle, en tant que voie réservée aux travailleurs ${ }^{8}$.»

Dès que l'approche de Gentile fut appliquée au champ de l'instruction artistique, il apparut nécessaire de l'épurer de tout caractère technique, «condition indispensable pour pénétrer le concept d'art $»^{9}$. C'est pourquoi il imagina, au sein de sa réforme du système scolaire, deux voies pour la formation artistique, recréant la vieille dichotomie entre les arts majeurs et les arts mineurs ${ }^{10}$ : les instituts d'art et les nouveaux lycées artistiques ${ }^{11}$.

5 Les instituts d'art devaient poursuivre l'objectif consistant à former au travail et à la production artistique, à fournir la culture nécessaire pour former des chefs d'atelier et à attirer les jeunes vers les travaux d'art appliqué ${ }^{12}$. Au contraire, les lycées artistiques, qui représentaient la voie la plus élitiste de la formation artistique, entendaient former l'artiste " pur ", au sein des huit Académie des Beaux-Arts qui existaient en Italie ${ }^{13}$. Le but étant de préparer aux études supérieures, on n'y enseignait pas les arts appliqués, mais l'art pur! Parmi les enseignements dispensés au lycée artistique, et au-delà des matières artistiques, furent introduits des enseignements de culture générale, comme la littérature italienne et étrangère, l'histoire et l'histoire de l'art, les mathématiques, la physique, l'histoire naturelle, la chimie et la géographie, enseignements pour lesquels, par ailleurs l'assiduité était obligatoire ${ }^{14}$.

6 En conformité avec le caractère très sélectif de la réforme de Gentile, l'article $17 \mathrm{du}$ décret royal du 31 décembre $1923\left(n^{\circ} 3123\right)$ prévoyait que, dans chacune des années du lycée artistique, on ne pouvait pas inscrire plus de cinquante élèves, choisis sur des critères de mérite ${ }^{15}$. Cette réforme basée sur l'idéal méritocratique était donc très classique $^{16}$ - ce qui, en soi, constituait une rupture avec les attentes du régime ${ }^{17}$ - et accusait de graves retards au regard des idées qui circulaient en Europe au cours des vingt premières années du XXe siècle. Elle privait en effet l'école de tout enseignement pratique, comme les travaux manuels ou les stages, mais aussi de sciences humaines comme la psychologie, la sociologie, la pédagogie «qui auraient pu perturber le triomphe pacifique, désormais reconnu, de l'idéalisme ${ }^{18} »$.

Un premier signe de crise entre la réforme de Gentile et le régime peut être observé à l'occasion du virage autoritaire du Duce, consécutif à la forte opposition à la démocratie et au parlementarisme du siècle précédent que mit en lumière l'assassinat de Giacomo Matteotti, le 10 juin $1924^{19}$.

\section{L'enseignement professionnel et l'art appliqué à l'industrie}

8 À partir de 1925, débarrassé de tout organe d'opinion indépendant, Mussolini s'attela à la construction progressive d'un État autoritaire et personnel. Le président du Conseil, qui jusque-là était considéré comme primus inter pares, fut transformé en chef du gouvernement, lequel n'était directement et individuellement responsable qu'envers le Roi. La nouvelle organisation de l'État conférait à Mussolini de nouvelles et importantes prérogatives en termes de nomination et licenciement des ministres, responsabilités 
qui incombaient auparavant au Roi. À partir de cette date, toute décision dut être approuvée par le Duce ${ }^{20}$.

Mussolini concentra ensuite ses efforts sur la réalisation d'une minutieuse campagne pédagogique en vue d'une ample diffusion de l'idéologie fasciste, basée sur une constante célébration de la culture du « chef », incarnation vivante de la nation, et sur l'exaltation des masses en vue de la création d'un homme nouveau et d'un nouvel ordre social ${ }^{21}$.

10 L'école héritée de l'État libéral, que Gentile avait restaurée sans la réformer, subit de nombreuses transformations qui avaient pour but de la fasciser. Giuseppe Belluzzo, en 1928 , réorganisa l'instruction technique et professionnelle, y compris dans des secteurs qui, dans le passé, dépendaient des ministères de l'Économie et passèrent dorénavant sous la dépendance du ministère de l'Instruction publique, afin de développer un enseignement qui soit en adéquation avec les besoins du monde de l'entreprise. En 1929, Giuliano Balbino inaugura le ministère de l'Éducation Nationale, dirigé entre 1935 et 1936 par Cesare Maria De Vecchi, lequel supprima toute forme d'autonomie de l'école en l'assujettissant totalement à l'État fasciste ${ }^{22}$.

11 C'est Giuseppe Bottai qui lui succéda à ce poste. Il était connu pour être sensible aux problématiques culturelles et considéré comme l'un des plus habiles intellectuels du régime ${ }^{23}$. Avec la Carte de l'école en 1939, il prévoyait «l'introduction du travail manuel dans toutes les branches et tous les niveaux de l'enseignement, avec une exaltation décisive de la technique au sein d'une école essentiellement humaniste et centrée sur le lycée » ${ }^{24}$. Avec Bottai, on se dirigea vers l'idée d'un art appliqué à l'industrie, dans le but de lier l'instruction artistique, tout spécialement dans les Instituts d'art, aux industries artistiques. L'organisation de l'instruction artistique, dans la Carte de l'école, fut encadré par les Dichiarazioni VIII ${ }^{25}$ et XX. La volonté du ministre de parvenir à repenser les rapports entre l'art pur et l'art appliqué est particulièrement claire dans la Dichiarazione XX, qui concerne l'organisation de l'instruction artistique :

«Les instituts d'art figuratif fondent intimement, dans leur organisation et leurs programmes, les techniques appliquées aux principes de l'art pur. Le cours d'initiation à l'art, pendant trois années, prépare aux travaux d'art élémentaires; l'École d'art, sur cinq années, prépare aux techniques de l'artisanat artistique; l'Institut d'art, sur huit ans, forme les maîtres d'art; le Cours supérieur pour le dessin et l'art appliqué, sur deux ans, habilite à l'enseignement de ces deux matières; le Lycée artistique, sur cinq ans, prépare à la formation technique et spirituelle des jeunes qui ont des aptitudes aux arts de la peinture et de la sculpture $[\ldots]^{26} »$.

12 En lien direct avec sa conviction personnelle selon laquelle le fascisme était «une révolution intellectuelle [...] qui s'emploie constamment à son propre perfectionnement et à sa réalisation, se situant entre les deux écueils d'une doctrine abstraite d'une part, et d'un pragmatisme empirique d'autre part, et dont l'objectif ultime, clair et précis, était de parvenir à achever la constitution d'un État organique national ${ }^{27}$ ", Bottai tenta de déconstruire le hiatus qui existait entre l' " art pur » des lycées et "l'art appliqué à l'industrie » des Instituts d'art, afin d'ouvrir la voie à la future instruction artistique. Le projet du ministre fut non seulement de former des artistes, mais aussi des jeunes capables d'exercer un métier et de les insérer dans la vie industrielle de la nation. Son orientation consista à libérer l'instruction artistique « de sa dimension abstraite et vaine $\aleph^{28}$, pour tendre à la formation globale de l'homme, en créant un lien entre les activités manuelles et les activités intellectuelles ${ }^{29}$. On 
demandait aux artistes d'être capables de conjuguer l'art et la technique, définissant ainsi un profil professionnel qui était en parfaite adéquation « avec le chemin politique et économique de la nation", loin de l'isolement de l'artiste envisagé comme un « génie » propre aux périodes du passé30.

Comme tous les autres secteurs culturels, l'art devait offrir sa contribution à la cause fasciste, tout spécialement dans les années de l'axe Rome-Berlin, lorsque l'écrasante supériorité industrielle allemande ne pouvait être contrebalancée que par la supériorité artistique et culturelle de l'Italie.

\section{L'éducation « globale et totalitaire » des masses : la contribution du théâtre et du cinématographe}

14 Les politiques de fascisation de l'école furent accompagnées par des actions de propagande vouées à la valorisation de l'éducation des masses en dehors des salles de classe $^{31}$, sous le contrôle des organes de l'État et du parti. L'organisation des masses, et non plus des classes, cet objet d'attention des anciens partis ${ }^{32}$, devint l'objectif principal de la politique fasciste ${ }^{33}$.

Ceci se traduisit par le besoin ressenti par le régime de « s'approprier les organisations sociales existantes, d'en créer de nouvelles, de multiplier la portée et les prérogatives des structures vers lesquelles diriger, depuis l'enfance, le plus possible d'hommes et de femmes ${ }^{34}$, comme l'Opera Nazionale Dopolavoro et l'Opera Nazionale Balilla, toutes deux directement dépendantes du Parti fasciste. L'Opera Nazionale Dopolavoro, créée par le Décret Royal du $1^{\text {er }}$ mai 1925 dans le but de prendre en charge le temps libre des travailleurs, devint rapidement le cadre central de l'enrégimentement des masses par le régime. Elle était présente sur tout le territoire national ${ }^{35}$. L'Opera Nazionale Balilla, créée en 1926, fut considérée comme «la véritable école du fascisme, non seulement parce qu'elle était gérée par des hommes totalement inféodés à la cause, mais surtout parce qu'elle incarnait l'idéal pédagogique des dirigeants en chemise noire ${ }^{36} »$.

16 La question du cadrage et de l'intégration des masses dans l'État donnait au régime l'occasion de tester sa propre capacité révolutionnaire à construire «une nouvelle civilisation politique » : l'éducation "globale et totalitaire » des masses, affirmait-on dans le quotidien Popolo d'Italia du 15 décembre 1929, constituait le problème central du fascisme ${ }^{37}$.

17 Au cours des années 1930, et tout spécialement au moment de la campagne d'Ethiopie (entre 1935 et 1936) ${ }^{38}$, l'action du parti fasciste s'orienta dans trois directions très précises :

18 «Vers la définition idéologique de l'État totalitaire, vers le développement systématique des formes d'organisation et d'enrégimentement des masses, sous la direction du PNF, pour un travail minutieux de formation au sens fasciste du terme, et vers la radicalisation du processus de concentration du pouvoir du fascisme, à travers une expansion croissante de la présence du parti dans la société et dans l'État, par une nouvelle série de réformes qui transformèrent substantiellement la vieille constitution du royaume ${ }^{39}$.

19 L'Opera Nazionale Dopolavoro absorba progressivement toutes les associations culturelles et sportives créées en Italie avant l'arrivée du fascisme, et constitua un instrument important de pénétration politique au sein des masses, tout spécialement après la mise 
en place du "Samedi fasciste ${ }^{40}$ ", un rendez-vous hebdomadaire à caractère pédagogique dédié à la formation de l'homme fasciste, présenté par les organes du Parti National Fasciste comme une des innovations les plus importantes du régime. Sur les terrains et dans les salles dédiées à l'instruction militaire et sportive, tous les citoyens, quelle que soit la classe sociale à laquelle ils appartiennent, devaient s'acquitter, volontairement et activement, de ce devoir fondamental qui consistait à y participer, conformément aux souhaits du régime.

Des activités à caractère strictement culturel commencèrent aussi à être organisées au sein des «Samedis fascistes", comme les visites guidées dans des sites archéologiques, dont certaines ont été filmées à l'époque par l'Institut National LUCE. Un de ces films montre les employés du ministère des Finances, accompagnés de leurs familles respectives, visitant un site archéologique, guidés par le professeur Ducci, dont les commentaires tendent à exalter la puissance, la grandeur et la civilisation de la Rome antique, en résonance avec le style fasciste ${ }^{41}$.

21 Le théâtre de masse fut ensuite introduit dans le cadre du "Samedi fasciste ${ }^{42}$ " grâce au renforcement des activités développées par les théâtres ambulants, les Carri di Tespi ${ }^{43}$, et les nombreuses compagnies de théâtre amateur qui intervenaient dans le cadre de l'opera Nazionale Dopolavoro. Le "Samedi théâtral » était une initiative rigoureusement réservée aux ouvriers et aux agriculteurs, aux vendeurs ambulants et aux commerçants, aux coursiers, aux huissiers, aux fonctionnaires, aux employés modestes en général dont les revenus mensuels ne dépassaient pas 800 lires, inscrits aux organisations de jeunesse, et bénéficiaires des œuvres d'assistance sociale du PNF. Ces spectacles rencontrèrent un grand succès public, grâce aux tarifs préférentiels pour les transports auxquels avaient accès les populations rurales. Le répertoire des Carri di Tespi $^{44}$ comportait, en majorité, des œuvres du théâtre traditionnel, sans objectif de propagande immédiat, mais avec l'intention de rassembler un vaste public.

En 1937, le ministère de la Culture Populaire, à la demande de Mussolini, encadra les activités existantes au sein d'un programme commun, l'Estate Musicale Italiana (l'Été musical italien), avec des spectacles réalisés dans 36 villes réparties sur le territoire national. Parmi ces manifestations, on peut tout particulièrement relever les représentations du «théâtre du peuple » aux Thermes de Caracalla à Rome et dans la cour principale du château Sforzesco de Milan.

Le secteur du théâtre attira rapidement l'attention de quelques dirigeants fascistes, l'instar de Roberto Farinacci, auteur de la pièce en trois actes Redenzione (1932), œuvre exempte de toute qualité artistique. Même Mussolini, en collaboration avec Giovacchino Forzano, écrivit quelques pièces de théatre, dont la plus connue, Campo di Maggio (1930), retrace les cent jours de Napoléon ${ }^{45}$. En 1931, le duo Mussolini-Forzano présenta tout d'abord Villafranca, œuvre dans laquelle Cavour est présenté comme plus puissant que le Roi, puis, en 1939, Cesare, qui exaltait de façon évidente la culture romaine et le Duce. Les deux pièces Campo di maggio et Villafranca rencontrèrent un certain succès, en Italie comme à l'étranger, et furent même adaptées au cinéma ${ }^{46}$.

Parallèlement aux œuvres des dirigeants, nombreuses furent les productions faisant l'apologie du régime et du Duce réalisées par des auteurs improvisés, complètement étrangers au monde du théâtre, comme Credere obbedire combattere ${ }^{47}$, Mussolini ci ha chiamato $^{48}$, Mussolinia, Spolverare il manganello ${ }^{49}$, Per il trionfo della civiltà fascista ${ }^{50}$, etc. Il s'agissait de productions peu intéressantes d'un point de vue artistique, mais qui 
rendaient compte d'une propagande du régime destinée à exalter les idéaux de force, courage et patriotisme.

Au-delà du théâtre, Mussolini eut aussi recours au cinématographe, une puissante machine à fabriquer du consensus politique ${ }^{51}$, qui fut tout d'abord mise sous le contrôle du ministère de la Presse et de la propagande (1935), puis du ministère de la Culture populaire (1937). L'Unione Cinematografica Educativa (L.U.C.E.) ${ }^{52}$, à laquelle avait été conféré le statut d'établissement public en 1925, se donnait pour mission « la diffusion de la culture populaire et de l'instruction générale par la voie des projections cinématographiques $»^{53}$. Chaque projection organisée par une salle de cinéma devait inclure au sein du programme un film éducatif, de propagande et culture ${ }^{54}$. Toutefois, dans le domaine de la fiction, les productions cinématographiques de propagande du régime ne rencontrèrent pas un grand intérêt de la part du public. Parmi les titres qui eurent le plus de succès, on peut noter : Camicia nera (G. Forzano, 1933), un film qui représentait les événements propres à l'histoire de l'Italie entre 1914 et 1932; Vecchia Guardia (A. Blasetti, 1933), qui glorifiait le squadrisme et la marche sur Rome; Lo squadrone bianco (A. Genina, 1936) et Scipione l'Africano (C. Gallone, 1937), deux films qui exaltaient la puissance colonisatrice de l'Italie ; et enfin 1860, (A. Blasetti, 1934), un film évoquant les liens entre le Risorgimento et l'avènement du fascisme.

\section{L'art envisagé comme un outil pédagogique au service du régime : la fusion entre la peinture, la sculpture et l'architecture dans les années trente}

La conviction fasciste selon laquelle l'éducation des masses devait être réalisée, en bonne partie, en dehors des salles de classe, sous le contrôle des organes d'État et du parti, se raccordait parfaitement à la volonté d'attribuer à l'art une fonction politique, éducative et sociale.

La politique culturelle du fascisme formula «un appel à la participation active, militante, des artistes à la cause du fascisme ${ }^{55}$ » afin qu'ils mettent leur talent au service de l'ordre politique en vigueur. Dans le discours prononcé le 26 mars 1923, à l'occasion de l'inauguration de l'exposition milanaise de la Galleria Pesaro, qui présentait les œuvres des artistes appartenant au nouveau groupe Novecento, Mussolini exprima sa volonté de poursuivre son projet politique avec la contribution de l'art et des artistes :

« On ne peut gouverner en ignorant l'art et les artistes; l'art est une manifestation essentielle de l'esprit humain; il est né en même temps que l'humanité et l'a accompagné jusqu'à aujourd'hui. Et dans un pays comme l'Italie, un gouvernement qui se désintéresserait de l'art et des artistes serait un gouvernent stupide. Je déclare que l'idée qui consisterait à encourager quelque chose qui pourrait ressembler à de l'art d'État m'est étrangère. L'art appartient au domaine de l'individu ${ }^{56} \%$.

Le fascisme ne développa pas à proprement parler un art d'État, et par voie de conséquence donna lieu à une esthétique variée. Dans le champ des arts visuels, des mouvements artistiques contrastés se sont côtoyés, comme les tendances avantgardistes qui célébraient le Duce ${ }^{57}$ et les tendances néoclassiques (que l'on retrouve dans les peintures murales "à l'antique » et les mosaïques, en particulier celles de Sironi), chapeautés par un mécénat d'État éclectique. Le style néo-classique se développa en lien avec le culte de la Rome impériale ${ }^{58}$, grâce auquel on pouvait 
développer des leçons morales et des exemples héroïques. Parmi les symboles fascistes récurrents, on peut relever la représentation des fasces ${ }^{59}$ portés par les littori ${ }^{60}$ de la Rome antique ${ }^{61}$, et l'exhumation de termes désuets comme "consul, cohorte, et centurion ». La vie devait être vécue - dans le nouveau jargon qui se généralisait - «à la romaine ${ }^{62} »$, et l'art pouvait fournir une contribution esthético-pédagogique importante pour frapper l'imaginaire du peuple italien.

Le secteur du bâtiment fut considéré par le régime comme un vecteur de publicité particulièrement avantageux, raison pour laquelle l'architecture bénéficia d'un intérêt particulier. Elle fut pensée de façon très originale, extravagante, et dans des proportions gigantesques, et fut utilisée sans scrupules, et sans qu'aucune contrainte ne lui soit imposée ${ }^{63}$. À Rome en 1924, Mussolini lança des opérations ambitieuses de fouilles qui, en l'espace d'une dizaine d'années, transformèrent la zone du Forum romain, mettant à jour tous les restes de l'époque de la Rome antique, au détriment des restes de l'époque des premiers siècles du christianisme ou du Moyen-Âge. Un exemple frappant de ces opérations de restyling ${ }^{64}$ fut le projet consistant à relier le Colisée à la place Venezia, par la route des Fori imperiali : avant 1932, entre les deux sites romains, on trouvait des quartiers issus de l'époque médiévale et la colline Velia, détruite pour construire la via dell'Impero. À l'extérieur de la basilique de Massenzio, «de grandes cartes géographiques en marbre furent apposées pour illustrer la grandeur de l'empire romain, de sorte à rappeler que ce que Rome avait fait une fois, on pouvait le refaire ${ }^{65}$ ». Se rattachant à la tendance artistique européenne néoclassique et traditionnaliste, les propositions du mouvement artistique Novecento, formé à l'initiative de l'intellectuelle Margherita Sarfatti, se prêtaient volontiers à la célébration des thèmes du passé, à l'évocation de la période antique, à la redécouverte de thématiques qui étaient devenues désuètes dans le climat de renouvellement artistique de ces années-là, comme la maternité (Maternità, A. Funi, 1921 ; Maternità, M. Sironi, 1923), la famille (Mio padre e le mie sorelle, A. Bucci, 1914), le sentiment patriotique et le rôle de la guerre (L'addio, A. Bucci, 1917 ; Vedove di guerra, L. Dudreville, 1916-1917). Le choix du nom du groupe, Novecento ${ }^{66}$, entendait conférer de l'importance au mouvement en l'ancrant historiquement à l'instar d'autres mouvements artistiques comme le Quattrocento et le Cinquecento ${ }^{67}$, afin de consacrer le siècle qui avait donné naissance à la conscience nationale, à l'italianité.

31 «En lien étroit avec les promesses fondatrices de 'Novecento' [...], on prôna la successivement la réalisation de fresques, de mosaïques et de reliefs décoratifs, au nom de la redécouverte de la décoration murale 'à l'antique'. Sironi, Funi, Campigli, Martini font partie de ceux qui contribuèrent le plus à la renaissance de ces expressions artistiques, considérées par les plus éminents théoriciens comme la manifestation privilégiée, et 'nécessaire', de la nouvelle époque fasciste ${ }^{68}$ ".

32 Au début des années 1930, Sironi et les muralistes se proposèrent de transformer la pratique artistique individuelle et 'bourgeoise' en une activité politique, à travers l'introduction de grandes fresques et décorations murales réalisées sur les façades et dans les atriums des espaces publics, pour que le peuple puisse en profiter pleinement. Les sujets privilégiés était le travail, la campagne (par exemple : La conquista della terra, D. Cambellotti, 1934, réalisée au Palazzo della Prefettura, à Latina), le commerce et l'activité industrielle, laquelle fut illustrée par le biais des grands travaux entrepris par le régime ${ }^{69}$. En décembre 1933, dans le Manifesto della pittura murale (Manifeste pour la peinture murale), Sironi exprime sa volonté de conférer «une fonction sociale et 
politique - donc liée à la polis - au peintre, appelé à maîtriser les surfaces et les matières premières des grands édifices publics ${ }^{70} »$. Selon Sironi, l'artiste doit :

«devenir un artiste 'militant', c'est-à-dire un artiste qui se met au service d'une idée morale, et qui subordonne sa propre individualité à l'œuvre collective [...]. La peinture murale est la peinture sociale par excellence. Elle opère sur l'imagination populaire plus directement que n'importe quelle autre forme de peinture, et inspire plus directement les arts mineurs. Le regain d'intérêt contemporain pour la peinture murale, et surtout pour la fresque, facilite la mise en place de l'Art Fasciste $^{71} \%$.

D'où la réunion de la peinture, la sculpture et l'architecture au cours des années 1930, cette dernière étant de toute évidence considérée comme un art d'État, au sein duquel la peinture et la sculpture occupaient une fonction complémentaire, en tant que supports idéologiques, pour rappeler le glorieux passé italien. Les sculptures décoratives à l'échelle monumentale s'intégraient au travail des architectes, avec des statues ou des bas-reliefs qui devaient «faire parler» les complexes architectoniques, par le biais de compositions rendant hommage aux liturgies politiques du régime et utiles à la création d'un nouvel imaginaire collectif. Selon Sironi, l'art était voué à remplir une fonction éducative au service de l'État fasciste, du fait même qu'on lui confiait le soin de produire le cadre éthique de la nouvelle nation.

L'art devait récupérer le rôle qu'il avait eu « dans ses périodes les plus fastes et au sein des plus grandes civilisations : un instrument parfait de gouvernance spirituelle ${ }^{72} »$. La mosaïque du Palais de la Justice de Milan célèbre la gloire de l'Italie en évoquant la romanité moderne du style propre au fascisme : «Ce n'est pas une imitation de Rome, mais une émulation de Rome, capable de dépasser Rome ${ }^{73}$ », y compris dans son caractère monumental.

La fresque réalisée dans la grande salle de la Città universitaria romana inaugurée en $1935^{74}$ a pour thème «L'Italie entre les arts et les sciences ». Elle est sévère, austère et monumentale. L'Italie est représentée au centre, surplombée par un majestueux aigle impérial, tandis qu'au fond sont disposés : une montagne rocheuse, dans laquelle est sculptée le fascio littorio avec l'indication de la date dans le calendrier fasciste, en l'occurrence l'année XIV, un arc de triomphe qui comporte un profil du Duce à cheval, et une Vittoria alata ${ }^{75}$ dynamique. Tous les arts et toutes les sciences entourent l'Italie fasciste, " pratiquement comme un chœur célébrant sa grandeur ${ }^{76}$ ».

\section{Conclusion}

36 À la fin des années 1930, la valeur politique de l'art dans le projet fasciste de l'Italie impériale fut renforcée par le ministre de l'Éducation nationale, Giuseppe Bottai, dans les pages du nouveau périodique Le Arti :

«L'État fasciste, dans sa doctrine, considère l'art comme un élément indispensable de l'éducation des masses. L'universalité de l'art italien, qui a conservé la suprématie de Rome malgré les infortunes politiques de l'Histoire, doit toujours être présent à l'esprit du peuple italien. [...] Je souhaite que la revue Le Arti offre au monde entier l'illustration du fait que l'art et la critique italienne sont totalement conscients de leur fonction, importante y compris d'un point de vue politique ${ }^{77}$ ».

37 Avec une approche culturelle différente par rapport à celle des hommes plus rustres du parti ${ }^{78}$, Giuseppe Bottai considérait que la répétition continuelle de slogans de propagande - pourtant régulière et encouragée - était peu efficace. Au contraire, il 
fallait selon lui s'appuyer sur le pouvoir de l'émotion et faire accéder les masses aux différentes expressions culturelles, depuis la peinture et l'architecture jusqu'au théâtre et au cinéma, afin de soutenir l'importance pour le nouvel homme italien de la stricte observance de la précieuse règle : «Croire ! Obéir ! Combattre !».

Aucun objectif strictement éducatif, émancipateur ${ }^{79}$, ne pouvait être rencontré au sein de cette propagande systématique opérée à l'aide des " produits culturels ", proposés à bas coûts ${ }^{80}$ pour être accessibles pour le peuple. Elle ne se préoccupait que d'exalter la figure du Duce, et de propager les aspirations et les buts de la révolution fasciste. Dans un cadre pédagogique uniformisant comme celui que dressa le régime fasciste, exempt de toute ouverture vers quelque forme que ce soit d'autonomie personnelle, on ne légitimait et n'autorisait que l'adéquation totale des masses avec les objectifs de la dictature. La valeur de chaque individu, identifié et évalué à l'aune de sa capacité à sacrifier son autonomie de jugement et de choix, transformait les hommes en véritables fascistes ${ }^{81}$.

\section{BIBLIOGRAPHIE}

Ainis, Michele ; Fiorillo, Mario. L'ordinamento della cultura: manuale di legislazione dei beni culturali. Milan, Giuffrè, 2008.

Ambrosoli, Luigi. Libertà e religione nella riforma Gentile. Firenze, Vallecchi, 1980.

Arendt, Hannah. Le origini del totalitarismo. Milan, Edizioni di Comunità, 1967.

Argentieri, Mino. L'occhio del regime. Informazione e propaganda nel cinema del fascismo. Florence, Vallecchi, 1979.

Ascenzi, Anna ; Sani Roberto (dir.). Il libro per la scuola tra idealismo e fascismo. Milan, Vita e Pensiero, 2005.

Bacigalupi, Marcella ; Fossati, Pietro. Da plebe a popolo. L'educazione popolare nei libri di scuola dall'Unità d'Italia alla repubblica. Florence, La Nuova Italia, 1986.

Bellucci, Maria ; Ciliberto, Michele. La scuola e la pedagogia del fascismo. Turin, Loescher, 1978. Betti, Carmen. L’Opera Nazionale Balilla e l'educazione fascista. Firenze, La Nuova Italia, 1984.

Bottai, Giuseppe. “Direttive del Ministero dell'Educazione Nazionale”, Le Arti, 1938-1939, n¹, octobre-novembre - Année I.

Bottai, Giuseppe. La carta della scuola. Milan, Mondadori, 1939.

Catalano, Franco. "La scuola italiana da Gentile a Bottai", in Italia contemporanea, 33, 1982, p. 126.

Cederna, Antonio. Mussolini urbanista: lo sventramento di Roma. Roma/Bari, Laterza, 1979.

Chiti, Edoardo ; Gardini Gianluca ; Sandulli, Aldo (dir.). Unità e pluralismo culturale Florence, FUP, 2016 
criscenti Grassi, Antonia. Progettare la formazione per i minori. Saggio di pedagogia critica. Catania, C.u.e.c.m., 2010.

De Angelis, Daniela (a cura di). Bottai e la mostra dell'istruzione artistica del 1939. Rome, Gangemi, 2008.

De Felice, Renzo (dir.). Il fascismo. Le interpretazioni dei contemporanei e degli storici. Roma/ Bari, Laterza, 1998.

Falasca Zamponi, Simonetta. Lo spettacolo del fascismo. Soveria Mannelli, Rubettino, 2003.

Galfré, Monica. Tutti a scuola!. Rome, Carocci, 2017.

Galfrè, Monica. Una riforma alla prova. La scuola media di Gentile e il fascismo. Milan, Franco Angeli, 2000.

Galli Della Loggia, Ernesto. Calendario. Un anno in Italia. Milan, 24 ORE Motta Cultura, 2008.

Gaudio, Angelo. Scuola, Chiesa e fascismo. Brescia, La Scuola, 1995.

Genovesi Giovanni. Storia della scuola in Italia. Dal Settecento ad oggi, Laterza, Roma-Bari 2004.

Gentile, Emilio. Il fascismo in tre capitoli. Roma/Bari, Laterza, 2004.

Gentile, Emilio. L’Italia tra le arti e le scienze di Mario Sironi. Rome/Bari, Laterza, 2014.

Gentile, Emilio. Le origini dell'ideologia fascista (1918-1925). Bologne, Il Mulino, 1996.

Gentili, Rino. Giuseppe Bottai e la riforma fascista della scuola. Florence, La Nuova Italia, 1979.

Insolera, Italo. Roma moderna: un secolo di storia urbanistica 1870-1970. Turin, Einaudi, 2001.

Janulardo, Ettore. “Sironi e il 'Novecento', la pittura murale”, Parol. Quaderni d'Arte e di Epistemologia, $\mathrm{n}^{\circ} 28,2017$, p. 3.

Lentini, Stefano. "Il 'Sabato fascista'. Un modello pedagogico dogmatico e omologante", Quaderni di Intercultura, X/2018, pp. 227-236.

Lentini, Stefano. "La fabbrica del consenso. Il contributo dell'arte negli anni del regime fascista", Annali della facoltà di Scienze della formazione, ${ }^{\circ} 18,2019$, pp. 49-65;

Luperini, Ilario. L'arte a scuola. Formazione artistica e riforma. Pisa, ETS, 2002.

Mack Smith, Denis. Storia d'Italia. Roma/Bari, Laterza, 2008.

Manetti, Daniela. Un' arma poderosissima. Industria cinematografica e Stato durante il fascismo 1922-1943. Milan, Franco Angeli, 2012.

Mele, Giorgio. Per la scuola di tutti. Breve storia della scuola italiana. Rome, Ediesse, 2014.

Montino, Davide. "Libro, quaderno e moschetto. Pedagogia della guerra nelle letture e nelle scritture scolastiche durante il regime fascista", in History of Education \& Children's Literature, II, 2007, n², pp. 193-216.

Nicoloso, Paolo. Mussolini architetto. Propaganda e paesaggio urbano nell'Italia fascista, Einaudi, Torino 2011.

Ostenc, Michel. La scuola italiana durante il fascismo. Rome/Bari, Laterza, 1981.

Pomante, Luigiaurelio. Giuseppe Bottai e il rinnovamento fascista dell'Università italiana (1936-1942), Franco Angeli, Milano 2017.

Ragazzini, Dario. "I programmi della scuola elementare durante il fascismo. Il caso dell'educazione linguistica”, in Orientamenti pedagogici, 1985, 32, pp. 1087-1117. 
Ricuperati, Giuseppe. La scuola italiana e il fascismo. Bologna, Consorzio Provinciale di Pubblica Lettura, 1977.

Roveda, Pietro. (dir.) Motivi pedagogici. In memoria di A. Leonarduzzi. Udine, Université de Udine, 1992.

Sacco, Domenico. "Fascismo e tempo libero: l'Opera nazionale dopolavoro", Eunomia. Rivista semestrale di Storia e Politica Internazionali, $n^{\circ} 1,2017$, p. 166.

Sarracino, Vincenzo ; Corbi, Enricomaria. Storia della scuola e delle istituzioni educative (1830-1999). Naples, Liguori, 2001.

Scarpellini, Emanuela. "Un palcoscenico per Mussolini: il Duce amava presentarsi come uomo di cultura, per questo scrisse opere teatrali in collaborazione con Giovacchino Forzano", Il Giornale, 11 janvier 2006.

Sironi Mario ; Carrà Carlo ; Funi Achille ; Campigli Massimo. "Manifesto della pittura murale”, La Colonna, Milan, 1933.

Tomasi, Tina. Idealismo e fascismo nella scuola italiana. Florence, La Nuova Italia, 1969.

visalberghi, Aldo. "Il fascismo e la scuola”, in Il Ponte, 1961, 17, pp. 691-701.

\section{NOTES}

1. Sarracino, Vincenzo. "Da Gentile alla Repubblica (1923-1946)”, in Sarracino, Vincenzo ; Corbi, Enricomaria. Storia della scuola e delle istituzioni educative (1830-1999). Naples, Liguori, 2001, pp. 53-54.

2. Catalano, Franco. "La scuola italiana da Gentile a Bottai", in Italia contemporanea, 33, 1982, p. 126. Voir aussi : Ostenc, Michel. La scuola italiana durante il fascismo. Rome/Bari, Laterza, 1981.

3. Parmi les pages que l'historiographie pédagogique italienne a dédié à l'histoire de l'école, on trouve de nombreux développements relatifs à la longue et controversée période fasciste. Voici quelques titres parmi les très nombreux travaux qui s'intéressent à l'histoire de l'école pendant cette période: Ambrosoli, Luigi. «Propaganda e proselitismo nei programmi e nei libri di testo della scuola durante il periodo fascista ", in Roveda, Pietro (dir.). Motivi pedagogici. In memoria di A. Leonarduzzi. Udine, Université de Udine, 1992 ; Ambrosoli, Luigi. Libertà e religione nella riforma Gentile. Firenze, Vallecchi, 1980 ; Ascenzi, Anna ; Sani Roberto (dir.). Il libro per la scuola tra idealismo e fascismo. Milan, Vita e Pensiero, 2005 ; Bacigalupi, Marcella ; Fossati, Pietro. Da plebe a popolo. L'educazione popolare nei libri di scuola dall'Unità d'Italia alla repubblica. Florence, La Nuova Italia, 1986 ; Bellucci, Maria ; Ciliberto, Michele. La scuola e la pedagogia del fascismo. Turin, Loescher, 1978 ; Galfrè, Monica. Una riforma alla prova. La scuola media di Gentile e il fascismo. Milan, Franco Angeli, 2000 ; Gaudio, Angelo. Scuola, Chiesa e fascismo. Brescia, La Scuola, 1995; Gentili, Rino Giuseppe Bottai e la riforma fascista della scuola. Florence, La Nuova Italia, 1979 ; Montino, Davide. “Libro, quaderno e moschetto. Pedagogia della guerra nelle letture e nelle scritture scolastiche durante il regime fascista", in History of Education \& Children's Literature, II, 2007, n², pp. 193-216 ; Ragazzini, Dario. "I programmi della scuola elementare durante il fascismo. Il caso dell'educazione linguistica", in Orientamenti pedagogici, 1985, 32, pp. 1087-1117; Ricuperati, Giuseppe. La scuola italiana e il fascismo. Bologna, Consorzio Provinciale di 
Pubblica Lettura, 1977 ; Tomasi, Tina. Idealismo e fascismo nella scuola italiana. Florence, La Nuova Italia, 1969 ; visalberghi, Aldo. “Il fascismo e la scuola”, in Il Ponte, 1961, 17, pp. 691-701.

4. Mele, Giorgio. Per la scuola di tutti. Breve storia della scuola italiana. Rome, Ediesse, 2014, p. 40.

5. Galfré, Monica. Tutti a scuola!. Rome, Carocci, 2017, p. 55.

6. Ibid., p. 43.

7. Ibidem.

8. Ibidem.

9. De Angelis, Daniela (a cura di). Bottai e la mostra dell'istruzione artistica del 1939. Rome, Gangemi, 2008, p. 39.

10. Luperini, Ilario. L'arte a scuola. Formazione artistica e riforma. Pisa, ETS, 2002, p. 73.

11. Décret Royal du 31 décembre 1923, n³123 : "Delle scuole ed istituti d'arte e degli istituti superiori per le industrie artistiche".

12. Ibid., articles 4-5.

13. Il s'agissait des établissements de Bologne, Florence, Milan, Naples, Palerme, Rome, Turin et Venise (Ibid., art. 13).

14. Les élèves inscrits dans chacune des années du lycée artistique avaient l'obligation de fréquenter les cours pour toutes les matières indiquées dans l'article 15. Ils pouvaient, cependant, sur la base d'une décision du Conseil des écoles, être exemptés de l'obligation de présence aux cours et aux examens pour trois matières de culture générale au plus, et être obligés, pour le nombre d'heures d'enseignement correspondant, à une plus forte présence dans un ou deux enseignements artistiques (art. 18).

15. À mérite égal, on préférait sélectionner le plus jeune des candidats.

16. Genovesi, Giovanni. Storia della scuola in Italia. Dal Settecento ad oggi. Rome/Bari, Laterza, 2004, p. 126.

17. Malgré l'importance de cette réforme de l'enseignement, Giovanni Gentile ne fut ministre de l'Instruction publique que pendant une très courte période, du 30 octobre 1922 au $1^{\text {er }}$ juillet 1924.

18. Catalano, Franco. Op. cit., p. 126.

19. C'est la raison pour laquelle Croce s'en éloigna, pour rejoindre l'opposition.

20. Mack Smith, Denis. Storia d'Italia. Roma/Bari, Laterza, 2008, p. 451.

21. Gentile, Emilio. Il fascismo in tre capitoli. Roma/Bari, Laterza, 2004, pp. 9-10.

22. Catalano, Franco. Op. cit., p. 128.

23. À propos de Bottai, on pourra consulter : Pomante, Luigiaurelio, Giuseppe Bottai e il rinnovamento fascista dell'Università italiana (1936-1942). Milano, Franco Angeli, 2017.

24. De Angelis, Daniela (dir.). Op. cit., p. 9.

25. Dans la VIII Dichiarazione, on peut lire: «Les Instituts dédiés à l'enseignement de l'art représentent des lieux spécifiques d'étude et de pratique, et s'organisent ainsi : 1. Cours d'initiation à l'art, trois ans; École d'art, cinq ans ; Institut d'art, huit ans; Enseignement supérieur pour le dessin et l'art appliqué, deux ans ; Lycée artistique, cinq ans ; Académie d'art, quatre ans [...] », (Bottai, Giuseppe. La carta della scuola. Milan, Mondadori, 1939, p. 79).

26. Ibid., p. 84.

27. Gentile, Emilio. Le origini dell'ideologia fascista (1918-1925). Bologne, Il Mulino, 1996, pp. 384-385.

28. Bottai, Giuseppe. "Intervista del Popolo d'Italia, 30 dicembre 1937-XVI", in Bottai, Giuseppe. La carta della scuola, op. cit., p. 242.

29. Ibid., p. 26.

30. Ibid., p.11. 
31. Catalano, Franco. Op. cit., p. 126.

32. Voir à ce propos : Arendt, Hannah. Le origini del totalitarismo. Milan, Edizioni di Comunità, 1967 ; Arendt, Hannah "Le origini del totalitarismo", in De Felice, Renzo (dir.). Il fascismo. Le interpretazioni dei contemporanei e degli storici. Roma/Bari, Laterza, 1998, p. 511.

33. Gentile, Emilio. La via italiana al totalitarismo. Il partito e lo Stato nel regime fascista, Op.cit., pp. 141-142.

34. Ibid., p. 141.

35. Sacco, Domenico. "Fascismo e tempo libero: l'Opera nazionale dopolavoro", Eunomia. Rivista semestrale di Storia e Politica Internazionali, $\mathrm{n}^{\circ} 1,2017$, p. 166.

36. Betti, Carmen. L'Opera Nazionale Balilla e l'educazione fascista. Firenze, La Nuova Italia, 1984, pp. 93-94.

37. Gentile, Emilio. La via italiana al totalitarismo. Il partito e lo Stato nel regime fascista. Rome, Carocci, 2002, p. 141.

38. Emilio Gentile observait : "Ce qui apparaît aujourd'hui évident pour celui qui observe la réalité du fascisme dans la seconde moitié des années trente, après le succès de la campagne d'Éthiopie, c'est l'accélération, volontaire et programmée, du processus tendant à conférer un caractère totalitaire à la société et à l'État » (Ibid., p. 137).

39. Ibidem.

40. Le "Samedi fasciste" fut créé par le Décret Royal du 20 juin 1935, n¹010, au cours de la session du Grand Conseil du 14 au 16 février 1935.

41. Archives de l'Institut National Luce, Journal LUCE B/B0714, 1935 [accessible en ligne] patrimonio.archivioluce.com/luce-web/detail/IL5000018767/2/il-sabatofascista-degli-impiegati-delministero- finanze.html (Consulté le 07/03/2020).

42. Voir le Décret Royal du 20 juin 1935, n¹010, qui crée le « samedi fasciste ».

43. Déjà en 1929, le régime, s'était servi des expériences de théâtre itinérant en plein air, en construisant quatre énormes structures théâtrales, trois pour le théâtre de prose, et une pour le théâtre lyrique, qui se déplaçaient à travers les provinces italiennes sur des camions dans le cadre de vastes tournées qui rassemblèrent des centaines de milliers de spectateurs. En janvier 1936 ont été expérimentées des représentations en matinée, dans le cadre du "Samedi fasciste ", à des prix particulièrement bas.

44. Thespis (VI ${ }^{\mathrm{e}}$ siècle av. J.C., dont aucune œuvre ne nous est parvenue, est considéré comme le fondateur de la tragédie en Grèce et symbolise la naissance du théâtre [note du traducteur].

45. Le nom de Mussolini n'apparaît jamais officiellement en tant que co-auteur dans l'édition italienne de l'œuvre, alors qu'il est mentionné dans la version française des Cent Jours.

46. Scarpellini, Emanuela. "Un palcoscenico per Mussolini: il Duce amava presentarsi come uomo di cultura, per questo scrisse opere teatrali in collaborazione con Giovacchino Forzano", Il Giornale, 11 janvier 2006.

47. Croire, obéir, combattre [note du traducteur].

48. Mussolini nous a appelés [note du traducteur].

49. Dépoussiérer la matraque [note du traducteur].

50. Pour le triomphe de la civilisation fasciste [note du traducteur].

51. Manetti, Daniela. Un' arma poderosissima. Industria cinematografica e Stato durante il fascismo 1922-1943. Milan, Franco Angeli, 2012, pp. 51-54.

52. Institut National LUCE [note du traducteur]. 
53. Cité par Argentieri, Mino. L'occhio del regime. Informazione e propaganda nel cinema del fascismo. Florence, Vallecchi, 1979, p. 19.

54. Comme prévu par le Décret Royal du 02/04/1926, n 1000. Voir aussi : Barbati, Carla "Lo spettacolo nella storia istituzionale italiana", in Chiti, Edoardo ; Gardini, Gianluca ; Sandulli Aldo (dir.). Unità e pluralismo culturale. Florence, FUP, 2016, p. 347.

55. Cf. Ainis, Michele ; Fiorillo, Mario. L'ordinamento della cultura: manuale di legislazione dei beni culturali. Milan, Giuffrè, 2008, pp. 49-50.

56. "Alla Mostra del Novecento. Parole di Mussolini sull'arte e sul governo", Il Popolo d'Italia, 27 mars 1923, cité par : Galli Della Loggia, Ernesto. Calendario. Un anno in Italia. Milan, 24 ORE Motta Cultura, 2008, p. 105.

57. Voir, par exemple : Aero- ritratto di Benito Mussolini aviatore (Ambrosi, Alfredo 1930) ou Aeroritratto Duce (Dottori, Gerardo 1933).

58. On pourra aussi se référer au texte de la conférence donnée à l'Istituto degli studi Roma, le 15 décembre 1938- XVII, intitulée Roma nella scuola italiana, publiée dans : Bottai, Giuseppe. La carta della scuola, Op. cit., pp. 256-266.

59. Faisceaux : à Rome, " assemblages de verges liées autour d'une hache, portés par les licteurs devant le titulaire d'une grande magistrature comme symbole de son autorité; emblème analogue du fascisme italien", Le Petit Robert de la langue française, 2020, p. 1006 [note du traducteur].

60. Licteur : à Rome, « officier public qui marchait devant les grands magistrats en portant une hache placée dans un faisceau de verges ", Le Petit Robert de la langue française, 2020, p. 1454, [note du traducteur].

61. Le Fascio Littorio était dans la Rome antique le symbole du pouvoir de l'État et de la justice, qui punit ou répare. Avec le Décret Royal du 12 décembre 1926, n²061, le Fascio Littorio dont on précise la forme officielle devient l'emblème de l'État fasciste en toutes circonstances.

62. Mack Smith, Denis. Op. cit., p. 480.

63. Cederna, Antonio. Mussolini urbanista: lo sventramento di Roma. Roma/Bari, Laterza, 1979 ; Nicoloso, Paolo. Mussolini architetto. Propaganda e paesaggio urbano nell'Italia fascista, Einaudi, Torino 2011 ; Insolera, Italo. Roma moderna: un secolo di storia urbanistica 1870-1970. Turin, Einaudi, 2001.

64. En anglais dans le texte.

65. Mack Smith, Denis. Op. cit., p. 480.

66. Novecento signifie littéralement « 900 », mais fait référence au $\mathrm{XX}^{\mathrm{e}}$ siècle, aux années 1900 [note du traducteur].

67. De la même façon, références aux XVe et XVI ${ }^{\mathrm{e}}$ siècle [note du traducteur].

68. Janulardo, Ettore. "Sironi e il 'Novecento', la pittura murale", Parol. Quaderni d'Arte e di Epistemologia, ${ }^{\circ} 28,2017$, p. 3.

69. Ibid., p. 4

70. On peut se référer, à ce propos, aux restructurations et transformations urbaines de la période 1936-1939. (Ibid., p. 6).

71. Sironi, Mario ; Carrà, Carlo ; Funi, Achille ; Campigli, Massimo. "Manifesto della pittura murale", La Colonna, Milan, 1933.

72. Ibidem.

73. Gentile, Emilio. L'Italia tra le arti e le scienze di Mario Sironi. Rome/Bari, Laterza, 2014, p. 9.

74. La fresque fut abîmée par un carton disposé en 1943 pour dissimuler l'œuvre. Elle fut repeinte, maladroitement, entre 1948 et 1950, pour occulter quelques symboles fascistes présents sur la peinture, et fut finalement récemment restaurée, en 2017, sur la base de son apparence originale. Ce qui en reste aujourd'hui est une fresque qui n'a plus rien à voir avec l'intention originelle de Sironi. (Gentile, Emilio. L'Italia tra le arti e le scienze di Mario Sironi, Op. cit., p. 2). 
75. La «victoire ailée » est une célèbre sculpture romaine représentant une femme ailée, devenue le symbole de la ville de Brescia [note du traducteur].

76. La « cité universitaire romaine » était destinée à accueillir l'Université de La Sapienza [note du traducteur].

77. Bottai, Giuseppe. “Direttive del Ministero dell’Educazione Nazionale”, Le Arti, 1938-1939, n¹, octobre-novembre - Année I.

78. Il suffit de penser à la grotesque Exposition «Anti-Lei » organisée par Starace en 1939, dans laquelle un espace avait été rempli de statues et d'images dédiées à la lutte contre l'utilisation du pronom révérencieux «Lei » [équivalent du vouvoiement en français - Note du traducteur] : les hommes du régime soutenaient le fait que les Romains ne connaissaient pas le «Lei» et que Dante utilisait seulement le «Voi » et le «Tu». Les Italiens de Mussolini devaient ignorer le «Lei », considéré comme « un triste produit d'importation ». En effet, comme le notait Umberto Eco : « Dans la Rome antique, on utilisait seulement le 'Tu', mais à l'époque impériale apparut un 'Vos' qui fut conservé ensuite pendant tout le moyen-âge (par exemple quand on s'adressait à un abbé) et dans la Divine Comédie apparait le 'Voi' quand on veut s'exprimer avec grand respect ('Siete voi, qui, ser Brunetto?'). L'usage du 'Lei' se développera au cours de la Renaissance, dans le vocabulaire de la chancellerie, et sous l'influence de l'espagnol» (Eco, Umberto. « Tu, Lei, la memoria e l'insulto, lectio magistralis ", intervention au Festival de la Communication, 14 septembre 2015).

79. Il s'agissait d'un modèle pédagogique exempt de dimension critique, qui ne cherchait aucunement à promouvoir chez l'individu une quelconque capacité à réagir face aux conditions sociales de son époque. Pour approfondir ces questions, voir : criscenti Grassi, Antonia. Progettare la formazione per i minori. Saggio di pedagogia critica. Catania, C.u.e.c.m., 2010.

80. Manetti, Daniela. Op. cit., p. 51.

81. Falasca Zamponi, Simonetta. Lo spettacolo del fascismo. Soveria Mannelli, Rubettino, 2003, p. 276.

\section{RÉSUMÉS}

Pour construire un consensus politique, le régime fasciste eut recours à l'art, à l'architecture et à tous les modes d'expression culturelle impliquant une dimension esthétique, comme le cinéma et le théâtre, dans le but d'éduquer le peuple en dehors de l'école. Dans le cadre des processus d'intégration et d'identification nationale, la crise du modèle éducatif de Gentile, qualifié de classique, ouvrait la voie à « l'école du travail» de Bottai. L'instruction artistique, tout d'abord vouée à l'art pur des Lycées artistiques de Gentile, s'est ensuite orientée vers l'art appliqué des Instituts d'art de la «Carte scolaire », poursuivant l'objectif de «rendre les instituts d'art plus proches de la vie », mais aussi et surtout des ambitions politiques du régime.

\section{INDEX}

Mots-clés : Art fasciste ; culture fasciste ; éducation esthétique ; instruction artistique ; pédagogie ; politique 
AUTEUR

\section{STEFANO LENTINI}

Stefano Lentini est docteur en « Fondamenti e metodi dei processi formativi ». Il est professore associato auprès du département des Sciences de l'éducation de l'Université de Catania (Italie). 